\title{
Association of the FGFR1 mutation with spontaneous hemorrhage in low-grade gliomas in pediatric and young adult patients
}

\author{
Yukitomo Ishi, MD, ${ }^{1}$ Shigeru Yamaguchi, MD, Kanako C. Hatanaka, MD, ${ }^{2}$ Michinari Okamoto, MD, ${ }^{1}$ \\ Hiroaki Motegi, MD, ${ }^{1}$ Hiroyuki Kobayashi, MD, ${ }^{1,3}$ Shunsuke Terasaka, MD, ${ }^{1,3}$ and \\ Kiyohiro Houkin, MD1,3
}

${ }^{1}$ Department of Neurosurgery, Hokkaido University School of Medicine, Kita-ku, Sapporo; 'Department of Surgical Pathology, Hokkaido University Hospital, Kita-ku, Sapporo; and 'Department of Neurosurgery, Kashiwaba Neurosurgical Hospital, Toyohiraku, Sapporo, Hokkaido, Japan

OBJECTIVE The authors aimed to investigate genetic alterations in low-grade gliomas (LGGs) in pediatric and young adult patients presenting with spontaneous hemorrhage.

METHODS Patients younger than 30 years of age with a pathological diagnosis of World Health Organization (WHO) grade I or II glioma and who had undergone treatment at the authors' institution were retrospectively examined. BRAF V600E, FGFR1 N546/K656, IDH1 R132, IDH2 R172, and KIAA1549-BRAF (K-B) fusion genetic alterations were identified, and the presence of spontaneous tumoral hemorrhage was recorded.

RESULTS Among 66 patients (39 with WHO grade I and 27 with grade II tumors), genetic analysis revealed $K-B$ fusion in $18(27.3 \%)$, BRAF V600E mutation in $14(21.2 \%), I D H 1 / 2$ mutation in 8 (12.1\%), and FGFR1 mutation in $4(6.1 \%)$. Spontaneous hemorrhage was observed in 5 patients (7.6\%); 4 of them had an FGFR1 mutation and 1 had K-B fusion. Univariate analysis revealed a statistically significant association of an FGFR1 mutation and a diencephalic location with spontaneous hemorrhage. Among 19 diencephalic cases including the optic pathway, hypothalamus, and thalamus, an FGFR1 mutation was significantly associated with spontaneous hemorrhage $(p<0.001)$. Four FGFR1 mutation cases illustrated the following results: 1) a 2-year-old female with pilomyxoid astrocytoma (PMA) harboring the FGFR1 K656E mutation presented with intraventricular hemorrhage (IVH); 2) a 6-year-old male with PMA harboring FGFR1 K656E and D652G mutations presented with intratumoral hemorrhage (ITH); 3) a 4-year-old female with diffuse astrocytoma harboring FGFR1 K656M and D652G mutations presented with IVH; and 4) a young adult patient with pilocytic astrocytoma with the FGFR1 N546K mutation presented with delayed ITH and IVH after 7 years of observation.

CONCLUSIONS Although the mechanism remains unclear, the FGFR1 mutation is associated with spontaneous hemorrhage in pediatric and young adult LGG.

https://thejns.org/doi/abs/10.3171/2019.12.JNS192155

KEYWORDS FGFR1; BRAF; hemorrhage; low-grade glioma; pediatric; young adult; oncology

$\mathrm{M}$ OST pilocytic astrocytomas (PAs) exhibit genetic alterations in $B R A F$, with either missense mutation of V600E or KIAA1549-BRAF $(K-B)$ fusion. ${ }^{2}$ Cerebellar hemisphere PAs typically harbor $K-B$ fusion, while both $B R A F$ V600E and $K-B$ fusion are frequent in supratentorial cases. ${ }^{2} K-B$ fusion is primarily observed in PA, ${ }^{31}$ whereas the BRAF V600E mutation is observed in PA as well as other low-grade gliomas (LGGs), such as ganglioglioma and pleomorphic xanthoastrocytoma. ${ }^{21}$
After $B R A F$ alterations, hotspot mutations of N546 or K656 in FGFRI are the second most common major genetic alteration in PA. ${ }^{10}$ FGFRI and BRAF participate in mitogen-activated protein kinase (MAPK) signaling..$^{10}$ An FGFR1 mutation as well as BRAF alterations cause activation of MAPK signaling, which drives the development of PA. ${ }^{10}$ Missense mutation and tyrosine kinase domain (TKD) duplication of FGFRI are also frequent in dysembryoplastic neuroepithelial tumor (DNT), pediatric oligo-

ABBREVIATIONS DA = diffuse astrocytoma; DNT = dysembryoplastic neuroepithelial tumor; $\mathrm{ITH}=$ intratumoral hemorrhage; $\mathrm{IVH}=$ intraventricular hemorrhage; $K$ - $B$ fusion = KIAA1549-BRAF fusion; $L G G$ = low-grade glioma; $M A P K=$ mitogen-activated protein kinase; $P A=$ pilocytic astrocytoma; $P C R=$ polymerase chain reaction; $P M A=$ pilomyxoid astrocytoma; TKD = tyrosine kinase domain; $\mathrm{WHO}=$ World Health Organization.

SUBMITTED August 10, 2019. ACCEPTED December 2, 2019.

INCLUDE WHEN CITING Published online February 14, 2020; DOI: 10.3171/2019.12.JNS192155. 
Ishi et al.

TABLE 1. Summary of pediatric and young adult LGGs with the FGFR1 mutation

\begin{tabular}{|c|c|c|c|c|c|c|c|}
\hline Case & Age at Onset (yrs) & Sex & Location & Pathology & Genetic Alternation & Type of Bleeding & Timing of Bleeding \\
\hline 1 & 2 & $\mathrm{~F}$ & Hypothalamus & PMA & FGFR1 p.K656E & IVH & Onset \\
\hline 2 & 6 & M & Thalamus & PMA & FGFR1 p.K656E + p.D652G & ITH & Onset \\
\hline 3 & 4 & $\mathrm{~F}$ & Hypothalamus & $\mathrm{DA}$ & FGFR1 p.K656M + p.D652G & IVH & Onset \\
\hline 4 & 19 & $M$ & Hypothalamus & PA & FGFR1 p.N546K & ITH + IVH & Delayed \\
\hline 5 & 15 & $\mathrm{~F}$ & Cerebellar & PA & $K-B$ (ex16_ex11) fusion & ITH & Onset \\
\hline
\end{tabular}

dendroglioma, and rosette-forming glioneuronal tumor. ${ }^{5,20}$ Therefore, an FGFRl mutation is important in pediatric and young adult LGGs, as is $B R A F$ alteration.

Spontaneous hemorrhage is a rare clinical event in patients with LGG, including PA..$^{22,27}$ Nonetheless, spontaneous hemorrhage can be lethal despite a benign tumor pathology; ${ }^{4,27}$ therefore, predictive risk factors should be determined. Little is known about tumor-specific genetic risk factors for spontaneous hemorrhage in LGG, despite recent progress in glioma genomic research. In this study, we identified a high incidence of FGFRl mutation in pediatric and young adult LGGs with spontaneous hemorrhage.

\section{Methods \\ Patient Population}

In this retrospective study, we included all patients younger than 30 years of age with World Health Organization (WHO) grade I and II gliomas who had undergone treatment at Hokkaido University Hospital between 2002 and 2019. Pathological diagnosis was based on the WHO Classification of Tumours of the Central Nervous System. Patient data including clinical course, treatment outcome, radiological imaging findings, and pathological findings were retrospectively analyzed. Institutional review board approval was obtained; as this study was retrospective, the requirement for informed consent was waived.

\section{Genetic Analysis}

DNA/RNA was extracted from frozen tumor tissue using the AllPrep DNA/RNA Mini kit (Qiagen) or from a formalin-fixed paraffin-embedded (FFPE) block using the ReliaPrep FFPE gDNA Miniprep system (Promega) and RNAstorm kit (Cell Data Sciences). First-strand cDNA was synthesized using a PrimeScript II 1st strand cDNA Synthesis kit (Takara) in accordance with the manufacturer's recommendations. Mutation hotspots at codon 132 of $I D H 1$, codon 172 of $I D H 2$, codon 600 of $B R A F$, and codon 546 of exon 12 and codon 656 of exon 14 of FGFRI were screened using Sanger sequencing. Genomic DNA was amplified by polymerase chain reaction (PCR) using Quick Taq HS DyeMix (Toyobo). The oligonucleotide primers used for PCR were as follows: forward primer for $I D H 1$, TGTGGAAATCACCAAATGGCAC; reverse primer for $I D H 1$, TACAAGTTGGAAATTTCTGGGC; forward primer for $I D H 2$, GGGAGCCCATCATCTGCA AAAA; reverse primer for IDH2, ACAAGAGGATGG CTAGGCGA; forward primer for $B R A F,{ }^{15}$ TGCTTGCTC TGATAGGAAAATG; reverse primer for $B R A F,{ }^{15}$ TGA
TGGGACCCACTCCAT; forward primer for FGFRI exon 12, CTTTAAAGCGGACGCAACA; reverse primer for $F G F R 1$ exon 12, GATACCCCAGCTCAGATCTTCTC; forward primer for FGFRl exon 14, AGGAATGTCCTG GTGACAGA; and reverse primer for FGFRI exon 14, CCACTCCTTGCTTCTCAGAT. Because almost all missense mutations in FGFRI occur in exon 12 or $14,,^{10,20}$ we performed mutation screening of FGFRI exclusively in these two exons. Cycle sequencing was conducted with the BigDye Terminator v3.1 Cycle Sequencing kit (Applied Biosystems) using forward and reverse PCR primers as sequencing primers. Sequencing data were obtained using the Genetic Analyzer 3130 Avant (Applied Biosystems). $K$ - $B$ fusion was screened by reverse transcriptase PCR using previously described primer sets. ${ }^{11}$

\section{Statistical Analysis}

Statistical analysis using Fisher's exact test was performed with EZR software (Saitama Medical Centre, Jichi Medical University, http://www.jichi.ac.jp/saitama-sct/ SaitamaHP.files/statmedEN.html; Kanda, 2012), which is a graphical user interface for R (version 2.13.0, R Foundation for Statistical Computing). A value of $p<0.05$ was considered statistically significant.

\section{Results}

\section{Spontaneous Hemorrhage in Pediatric and Young Adult LGGs}

Sixty-six patients (39 with WHO grade I and 27 with grade II tumors) were included in this study. Five (7.6\%) developed spontaneous hemorrhage during the follow-up period; 4 of these cases presented acutely, and 1 presented in a delayed fashion (Table 1). The frequency of spontaneous hemorrhage was $5.1 \%$ and $11.1 \%$ in WHO grade I and II tumors, respectively. Location of the tumor was midline, including the hypothalamus and thalamus, in 4 cases. Genetic analysis revealed an FGFRI mutation in 4 cases and $K-B$ fusion in 1 case. Because an FGFRl mutation was frequent in the hemorrhagic cases, we present 4 such cases below.

\section{Case Presentations}

\section{Case 1}

This female patient was initially treated for intraventricular hemorrhage (IVH) at the age of 2 years (Fig. 1A). Since then, she has been bed-ridden with severe neurological deficits. Although MRI was not performed at the 

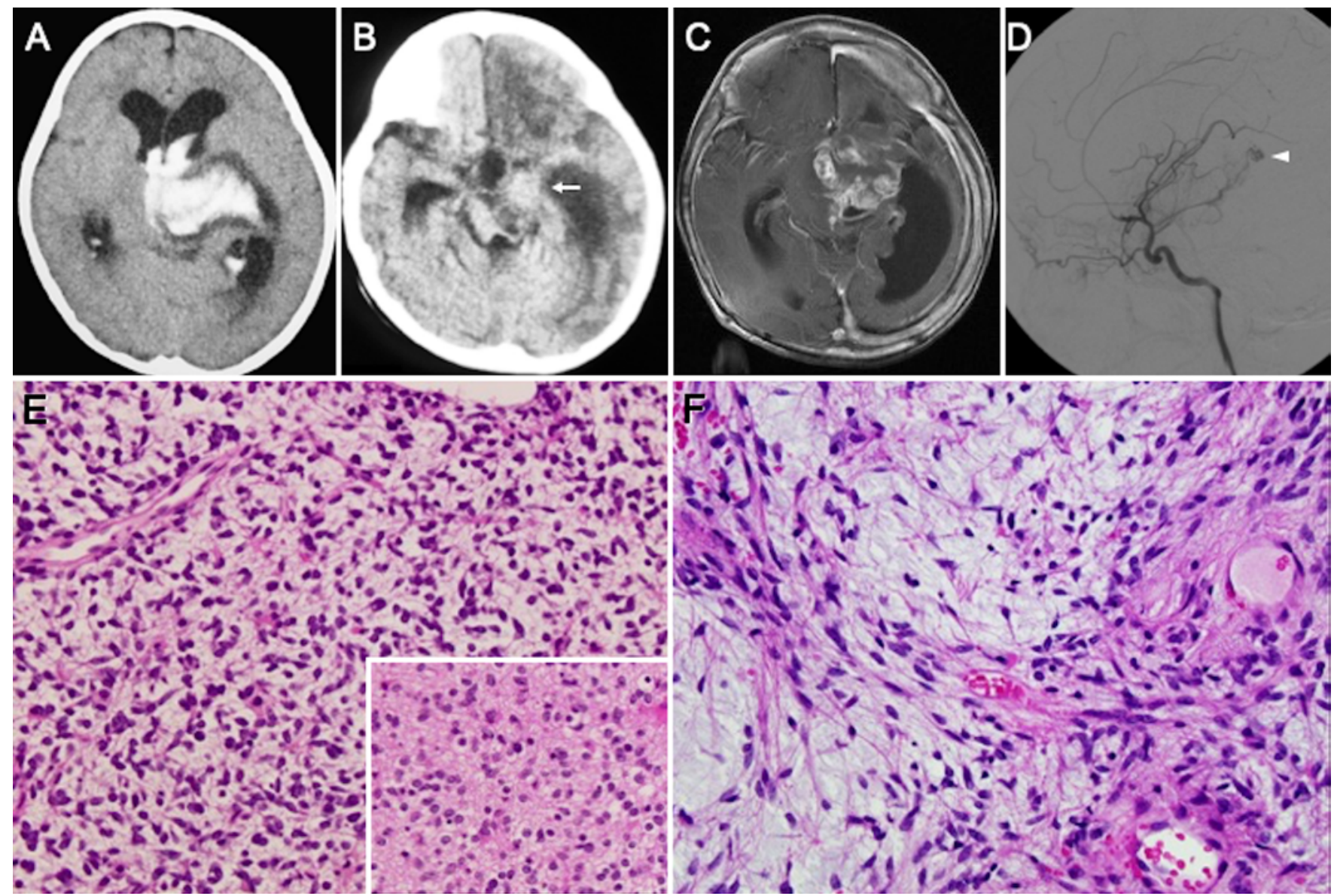

FIG. 1. Case 1. A: Head CT obtained when the patient was 2 years of age, showing IVH. B: Head CT obtained 2 months after hemorrhage, revealing cerebral infarction in the left hemisphere and a mass lesion in the left hypothalamic region (arrow). C: Gd-enhanced T1-weighted MR image obtained at age 4 years, showing tumor with heterogeneous enhancement in the left hypothalamus. D: Left carotid artery DSA revealed abnormal vasculature fed by the anterior choroidal artery. E: $\mathrm{H} \& \mathrm{E}$ staining of the biopsy specimen at the first operation revealed a pathological diagnosis of anaplastic astrocytoma with densely proliferating astrocytic tumor cells and oligodendroglial tumor cells (inset). Original magnification $\times 10$. F: $\mathrm{H} \& \mathrm{E}$ staining of the specimen at the second operation due to regrowth of residual tumor revealed a pathological diagnosis of PMA, based on a pilomyxoid background and angiocentric pattern. Original magnification $\times 20$.

time, serial CT revealed a mass lesion in the posthemorrhagic cavity as the hemorrhage had resolved (Fig. 1B). At the age of 4 years, she was referred to our institution because of a newly observed tumor at the hemorrhage site. MRI revealed a tumor in the left hypothalamus (Fig. 1C), and DSA revealed abnormal vessels supplied from the left anterior choroidal artery (Fig. 1D). She underwent surgical biopsy, and the pathological diagnosis was anaplastic oligoastrocytoma (Fig. 1E). She received chemotherapy consisting of a single course of cisplatin (CDDP) and etoposide (VP-16), followed by a course of carboplatin (CBDCA) and VP-16 and radiotherapy (25.2 Gy of whole craniospinal radiation and 25.2 Gy of local radiation). She then underwent another resection for residual tumor; the pathological diagnosis was pilomyxoid astrocytoma (PMA; Fig. 1F). Because the first surgical specimen was very small for pathological diagnosis and the pathological findings of the second surgical specimen were typical for PMA, we believe that the pathological findings of the first specimen were part of the whole tumor pathology; therefore, PMA was established as the final diagnosis. No vascular abnormality was identified in either the first or second surgical specimen. The patient underwent 2 additional courses of chemotherapy with CBDCA and vincristine (VCR); however, she died at the age of 5 years as a result of local progression and dissemination of the tumor. Genetic analysis showed the FGFRl K656E mutation in both the biopsy specimen and the resective operation.

\section{Case 2}

This male patient presented with the sudden onset of headache and vomiting at the age of 6 years. Because brain MRI showed an intracranial tumor, he was referred to our institution. Preoperative CT and MRI showed intratumoral hemorrhage (ITH) in the suprasellar region (Fig. 2A-B). DSA revealed no abnormal vascular structures (Fig. 2C). He underwent emergent surgery for tumor resection. The pathological diagnosis was PMA (Fig. 2D), and genetic analysis showed FGFR1 D652G and K656E mutations. No vascular abnormality was pathologically 

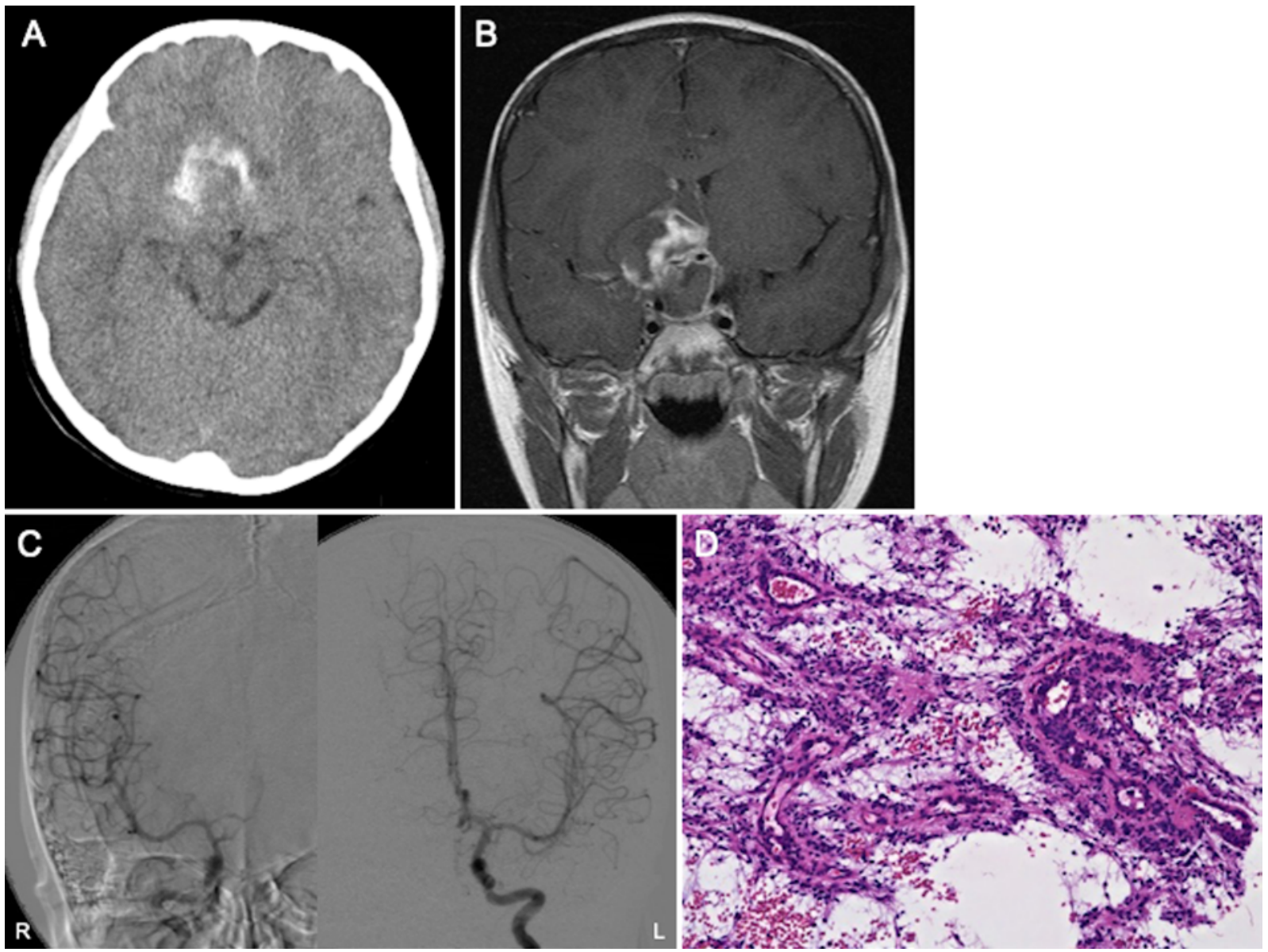

FIG. 2. Case 2. A: Initial head CT showing a high-density mass in the suprasellar region. B: Gd-enhanced T1-weighted MR image showing tumor with heterogeneous enhancement in the right hypothalamus. C: Bilateral carotid artery DSA $(R=$ right, $L=l e f t)$ demonstrating no apparent vascular abnormality. D: H \& E staining of the tumor specimen revealed a pathological diagnosis of $\mathrm{PMA}$, based on the pilomyxoid background and angiocentric pattern. Original magnification $\times 20$.

identified. He underwent 6 courses of chemotherapy with CDDP and VCR and local radiation with 50.4 Gy. He remains alive without neurological deterioration or tumor recurrence at 11 years.

\section{Case 3}

This female patient was referred to another hospital due to sudden headache and vomiting at the age of 4 years. Head CT showed IVH and she underwent ventricular drainage (Fig. 3A). She was then referred to our institution after MRI showed a brain tumor in the left thalamus (Fig. 3B). Stereotactic biopsy of the tumor was performed. The pathological diagnosis was diffuse astrocytoma (DA; Fig. 3C), and genetic analysis showed FGFRI D652G and K656M mutations. No vascular abnormality was pathologically identified. She underwent local radiation with $46 \mathrm{~Gy}$. She remains alive without neurological deficit or tumor recurrence at 19 years.

\section{Case 4}

This 19-year-old male presented with visual disturbance in his right eye. MRI showed a suprasellar tumor (Fig. 4A), and he underwent craniotomy for partial re- section. The pathological diagnosis was PA, and genetic analysis revealed the FGFRI N546K mutation (Fig. 4B). No vascular abnormality was pathologically identified. Considering his age, the patient was observed and not provided adjuvant treatment. Although the tumor showed repeated growth and shrinkage on serial imaging, he did not develop a neurological deficit over about 6 years after surgery. However, he then presented with coma 2 months after the last MRI study. Head CT showed massive ITH and IVH (Fig. 4C). Three-dimensional CTA showed no evidence of vascular abnormality (Fig. 4D). The patient underwent emergent ventricular drainage but remains comatose.

\section{Genetic Landscape and Spontaneous Hemorrhage in Pediatric and Young Adult LGGs}

Sixty-six patients underwent genetic analysis for $B R A F$, FGFRI, and $I D H I / 2$ (Fig. 5). $K$ - $B$ fusion was detected in 18 patients (27.3\%), and the BRAF V600E mutation was detected in $14(21.2 \%)$, frequently in WHO grade I gliomas. The IDHI/2 mutation was detected in 8 patients (12.1\%), all in WHO grade II gliomas. An FGFRI mutation was detected in 4 patients (6.1\%). Spontaneous hem- 
Ishi et al.
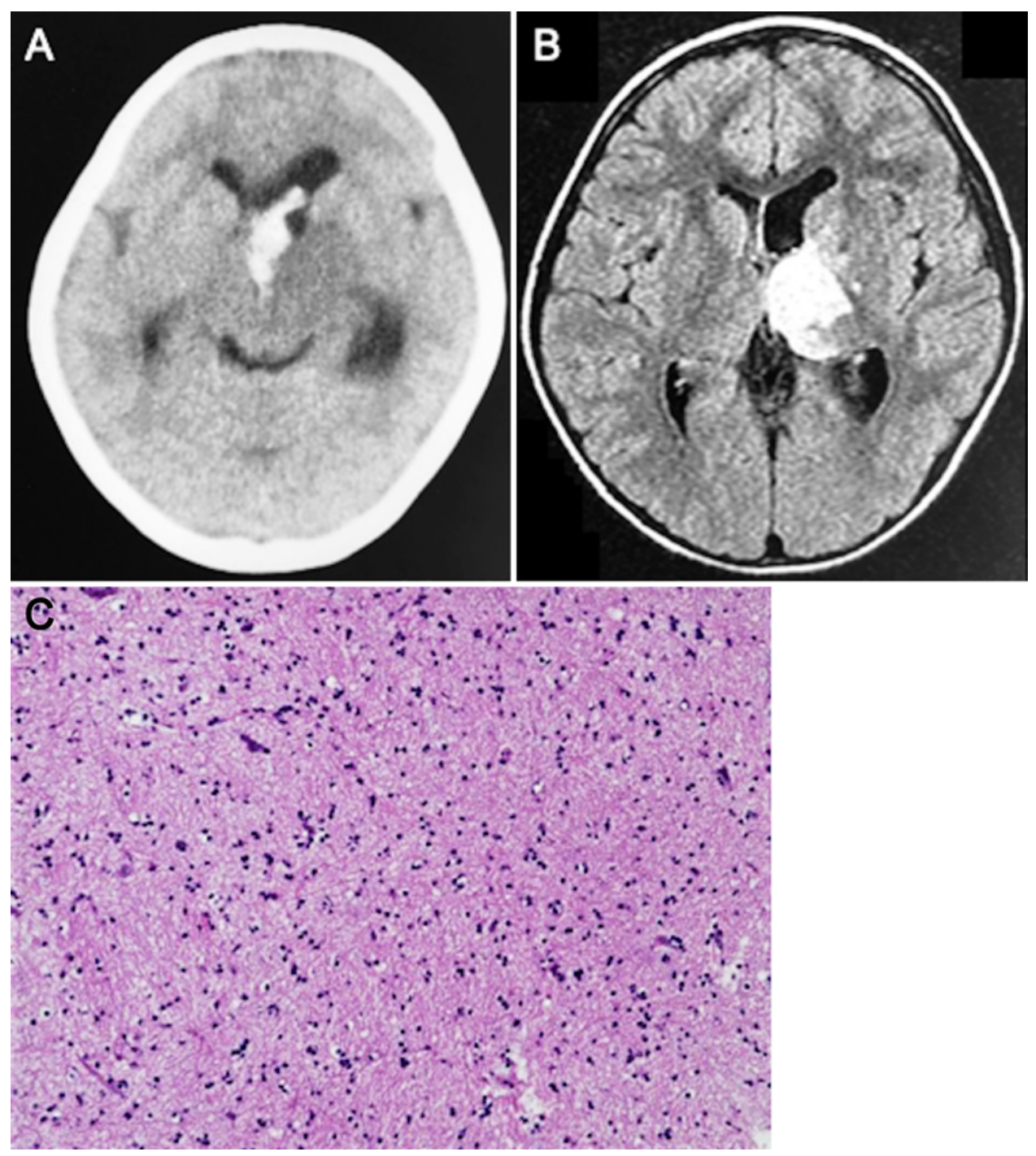

FIG. 3. Case 3. A: Initial head CT showing IVH. B: FLAIR MR image showing tumor with high intensity in the left thalamus. C: $H$ \& E staining of the biopsy specimen revealed a pathological diagnosis of DA, based on diffusely invading astrocytic tumor cells. Original magnification $\times 20$.

orrhage was observed in $55.6 \%$ of patients with $K-B$ fusion and in $100 \%$ of those with FGFRI mutations. None of the patients with the $B R A F$ mutation, the $I D H$ mutation, or wild-type BRAF/FGFRI/IDH presented with spontaneous hemorrhage. Univariate analysis revealed a statistically significant association of an FGFR1 mutation and a diencephalic location with spontaneous hemorrhage (Table 2). Among 19 diencephalic cases including the optic pathway, hypothalamus, and thalamus, an FGFRI mutation was significantly associated with spontaneous hemorrhage ( $\mathrm{p}<0.001$; Table 3$)$.

\section{Discussion}

\section{Clinical Characteristics of LGGs With an FGFR1 Mutation}

Previous reports have indicated that the incidence of an FGFRl mutation is $5.2 \%-6.7 \%$ in $\mathrm{PA}, 1,1036.4 \%$ in DNT, ${ }^{20} 10 \%$ in oligodendroglioma, ${ }^{20}$ and $46.2 \%-50 \%$ in rosette-forming glioneuronal tumor. ${ }^{5,14}$ Our results suggest that FGFRI mutation is also observed in pediatric and young adult cases of DA and PMA. All 4 cases harboring an FGFRI mutation in our study were located in the thalamus and hypothalamus, which is consistent with previous reports of PA. ${ }^{10}$ Although the FGFR1 mutation is associated with a poor prognosis in PA, ${ }^{1}$ our cases had a significantly different outcome, suggesting clinical heterogeneity with respect to the FGFRI mutation.

Despite the fact that the FGFRI mutation has been observed in several types of LGG, the pathological findings of case 1 were unusual. Previous studies have suggested that PA and PMA lacking the classic pathological features could be diagnosed as several other types of tumors., ${ }^{2,7}$

\section{Spontaneous Hemorrhage in LGGs}

Spontaneous hemorrhage has been observed in $8.0 \%-$ $24.0 \%$ of PA and PMA cases. ${ }^{16,22,28}$ Histologically, a high 

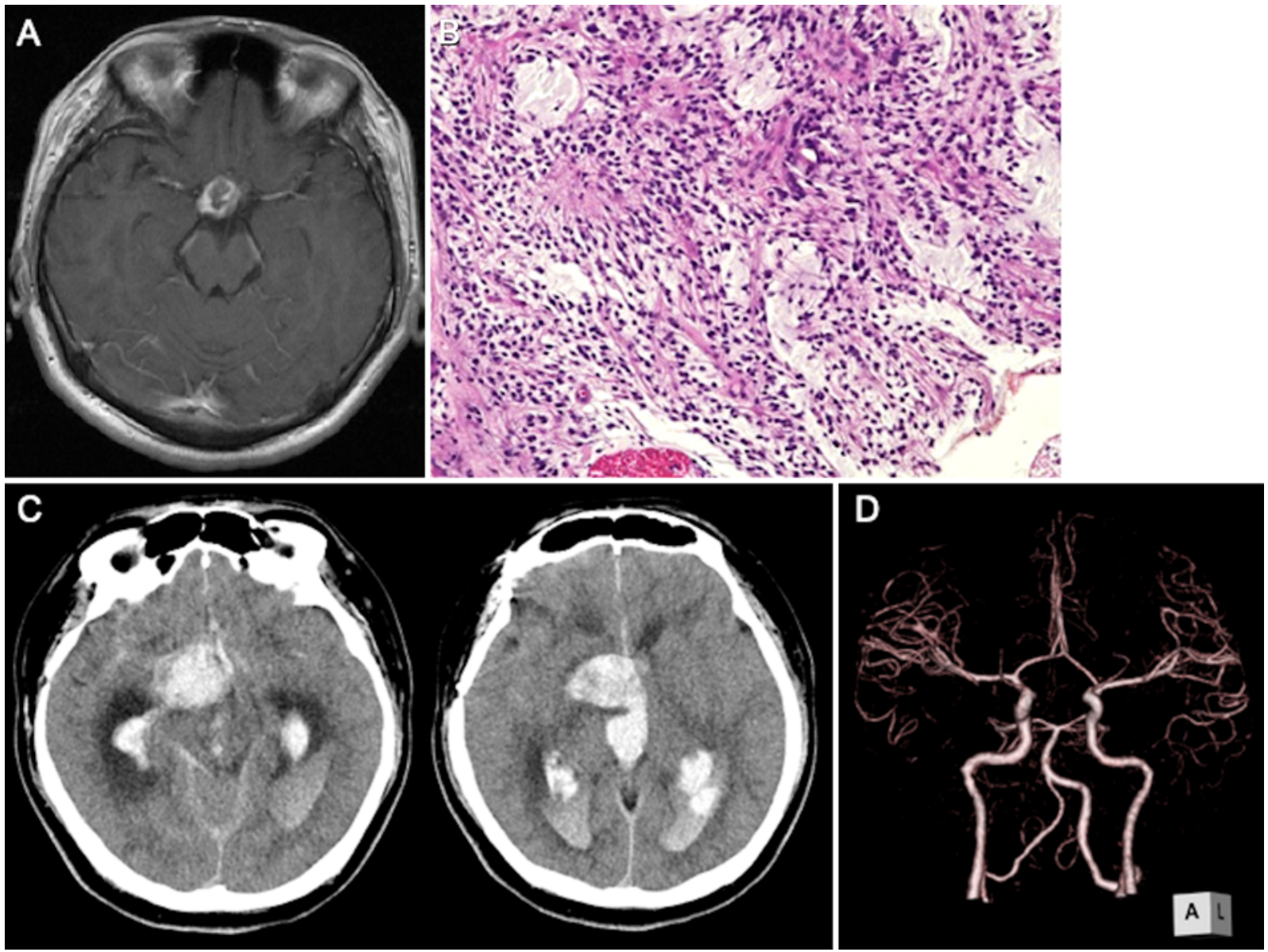

FIG. 4. Case 4. A: Preoperative MR image obtained when the patient was 19 years old, showing suprasellar tumor. B: $\mathrm{H} \& \mathrm{E}$ staining of the biopsy specimen revealed a pathological diagnosis of PA, based on a myxoid background and biphasic pattern with sparsely accumulating piloid tumor cells and densely accumulating tumor cells. Original magnification $\times 20$. C: Head CT scans obtained when the patient was 26 years old, showing massive ITH and IVH. D: Three-dimensional CTA showed no evidence of vascular abnormality.

frequency of hemorrhage in PMA cases has been reported. ${ }^{12,16}$ Although several risk factors for hemorrhage have been described, a recent large study on optic pathway-hypothalamic gliomas with spontaneous hemorrhage could not establish any risk factors. ${ }^{27}$ In addition, a review of cerebellar PAs with hemorrhage did not clarify the pathophysiology of hemorrhage. ${ }^{4}$

Previous authors have reported that $2(5.9 \%)$ of 34 optic pathway gliomas with hemorrhage have occurred in pregnant women..$^{27,30}$ Because no cases of pregnancy were included in the present study, it is not certain whether pregnancy is a risk factor for spontaneous hemorrhage in gliomas. Moreover, degenerative vascular changes, abnormal vasculature, or amyloid vessels have been reported as causes of spontaneous hemorrhage, ${ }^{17,23,28}$ but these factors were not applicable in our study as no hemorrhagic cases presented with pathological vasculature abnormality. Previous reports have stated that the absence of pathological findings indicative of spontaneous hemorrhage is not uncommon. ${ }^{6,13,19,24}$ As regards angiographic findings, Suzuki et al. have reported the case of a diencephalic PA presenting with no vascular abnormality on DSA..$^{25}$ Although 1 case in this study presented with vascular abnormality on DSA, such cases would be relatively rare among hemorrhagic cases. ${ }^{27}$

To the best of our knowledge, no previous studies have described an association between spontaneous hemorrhage and specific genetic alterations in a large number of patients. Wilson et al. have reported 2 cases of cerebellar PA in which genetic analysis was performed for $B R A F$ V600E mutation and $K-B$ fusion; however, these genetic alterations were not detected in these 2 cases. ${ }^{29}$ Although a large number of patients with LGG such as PA presenting with hemorrhage have been reported, ${ }^{19,27}$ most patients have not undergone genetic analysis. This study shows a correlation between the FGFRI mutation and spontaneous hemorrhage in LGGs in pediatric and young adult patients. Our data also indicated the predominance of hemorrhage in diencephalic cases, which has not been previously reported in the literature. As FGFRI-mutated PAs frequently arise in midline locations, including the diencephalon, ${ }^{10}$ we consider that the predominance of hemorrhage in diencephalic cases and FGFRl mutation would be confounding factors in this study. 


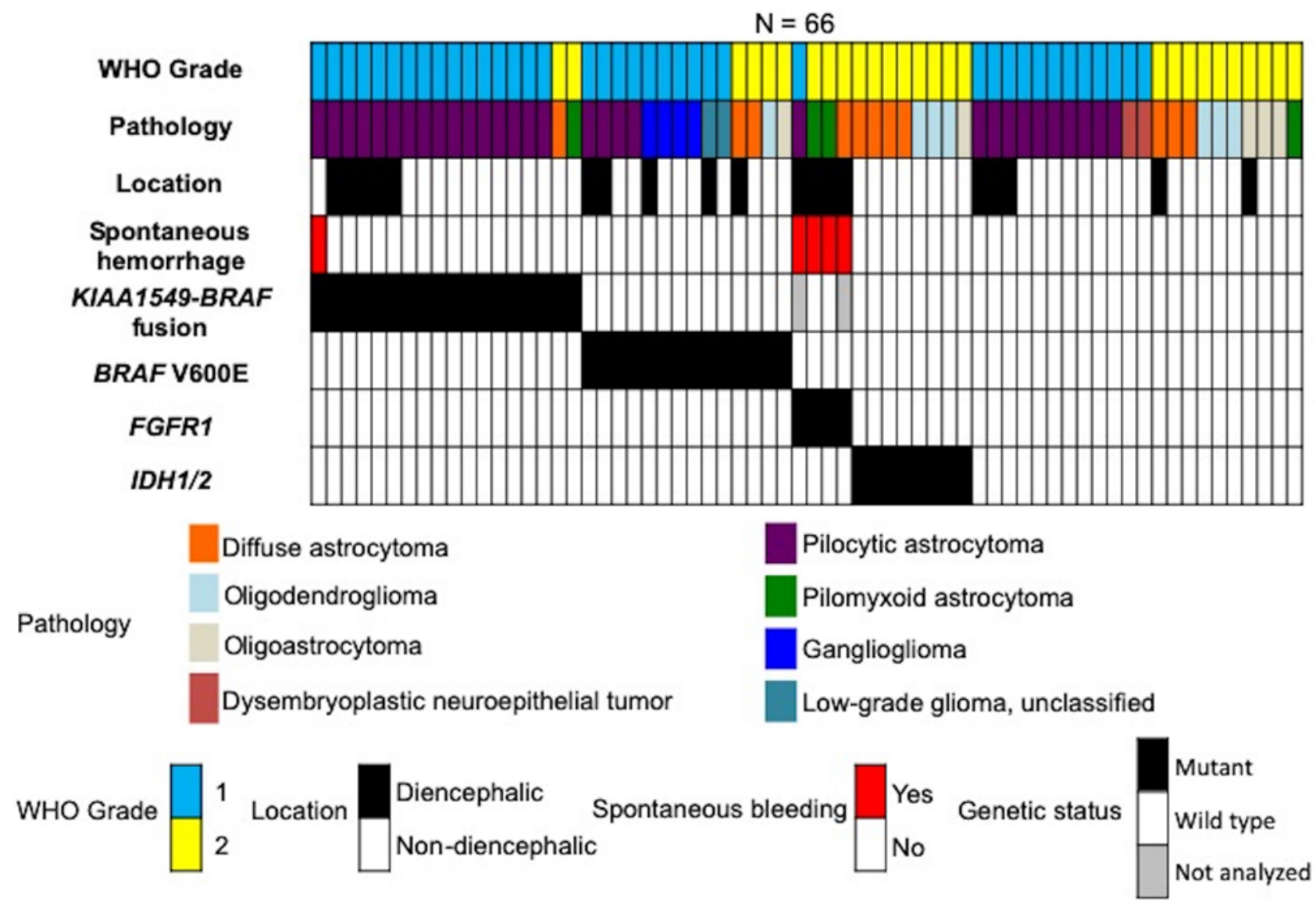

FIG. 5. Spontaneous hemorrhage and genetic landscape of LGGs. Spontaneous hemorrhage was observed in 5 cases of LGG, including 4 harboring an FGFR1 mutation and 1 harboring $K-B$ fusion.

\section{Other Genetic Alterations in FGFR1}

Genetic alterations in TKD duplication or missense mutation in FGFRl have been frequently reported in DNTs. ${ }^{20}$ Although several cases of DNT with spontaneous hemorrhage have been described, ${ }^{26}$ previous studies have demonstrated a relatively low frequency, with $2 \%-6.3 \%$ of hemorrhage cases in DNT. ${ }^{3,18}$ As our study included only

TABLE 2. Univariate analysis of associations with spontaneous hemorrhage using Fisher's exact test

\begin{tabular}{cccc}
\hline Factor & OR & $95 \% \mathrm{Cl}$ & $\mathrm{p} \mathrm{Value}$ \\
\hline Age $<15$ yrs & 1.04 & $0.11-13.31$ & 0.99 \\
\hline Location & & & \\
\hline Supratentorial & 1.80 & $0.16-93.69$ & 0.99 \\
\hline Midline & 6.43 & $0.59-333.18$ & 0.15 \\
\hline Diencephalic & 12.67 & $0.002-0.95$ & $<0.05$ \\
\hline PA/PMA pathology & 3.80 & $0.35-196.7$ & 0.36 \\
\hline Genetic alteration & & & \\
\hline K-B fusion & 1.54 & $0.14-80.49$ & 0.99 \\
\hline FGFR1 & $\operatorname{Inf}$ & $0.00-0.17$ & $<0.000001$ \\
\hline
\end{tabular}

Inf $=$ infinite.

Boldface type indicates statistical significance $(p<0.05)$.
1 case with DNT, the correlation between genetic alteration and hemorrhage in DNT was uncertain. In diencephalic LGGs harboring the $B R A F$ alteration of $K$ - $B$ fusion or V600E mutation, clinical differences between these groups have been reported despite the genetic alterations in the same gene., 70 the best of our knowledge, no previous study has analyzed the association between genetic alterations and hemorrhage in DNT; therefore, further investigation on TKD duplication and missense mutation in FGFRl is warranted for DNTs.

\section{Genotype-Phenotype Correlation of FGFR1 Mutation and Spontaneous Hemorrhage}

The mechanism regarding how the FGFRI mutation re-

TABLE 3. Correlation of FGFR1 status and spontaneous bleeding among 19 diencephalic cases

\begin{tabular}{ccc}
\hline & \multicolumn{2}{c}{ Spontaneous Bleeding } \\
\cline { 2 - 3 } FGFR1 Mutation & + & - \\
\hline Mutant & 4 & 0 \\
\hline Wild type & 0 & 15 \\
\hline$+=$ yes; $-=$ no. \\
$p<0.001$, Fisher's exact test.
\end{tabular}


sults in spontaneous hemorrhage is unclear. Radiological and pathological examinations did not suggest the presence of abnormal vessels that could cause spontaneous hemorrhage. Shibahara et al. reported that Ki-67 labeling index and microvascular proliferation were not statistically different between hemorrhagic and nonhemorrhagic cases of PA. ${ }^{22}$ Activation of the MAPK signaling pathway is commonly observed in LGGs such as PA. ${ }^{9}$ Because FGFR1 acts as an upstream protein to BRAF in MAPK signaling, ${ }^{10}$ we speculate that other effects in the signaling pathway due to an FGFRI mutation cause a biological difference compared with that in $B R A F$-altered cases. We believe that the presence of potential confounding factors must also be considered in this study. To confirm the significance of an FGFRI mutation in spontaneous hemorrhage, validation with a study involving a larger number of patients would be required.

\section{Conclusions}

Although the mechanism remains unclear, the association of an FGFRl mutation with spontaneous hemorrhage in pediatric and young adult LGG is suggested based on the results of this study. Because the specific mechanism remains unclear, the molecular function of an FGFRl mutation for tumor hemorrhage should be clarified.

\section{Acknowledgments} review.

We would like to thank Enago for the English language

\section{References}

1. Becker AP, Scapulatempo-Neto C, Carloni AC, Paulino A, Sheren J, Aisner DL, et al: KIAA1549: BRAF gene fusion and FGFR1 hotspot mutations are prognostic factors in pilocytic astrocytomas. J Neuropathol Exp Neurol 74:743-754, 2015

2. Collins VP, Jones DT, Giannini C: Pilocytic astrocytoma: pathology, molecular mechanisms and markers. Acta Neuropathol 129:775-788, 2015

3. Daghistani R, Miller E, Kulkarni AV, Widjaja E: Atypical characteristics and behavior of dysembryoplastic neuroepithelial tumors. Neuroradiology 55:217-224, 2013

4. Donofrio CA, Gagliardi F, Callea M, da Passano CF, Terreni MR, Cavalli A, et al: Pediatric cerebellar pilocytic astrocytoma presenting with spontaneous intratumoral hemorrhage. Neurosurg Rev [epub ahead of print], 2018

5. Gessi M, Moneim YA, Hammes J, Goschzik T, Scholz M, Denkhaus D, et al: FGFR1 mutations in Rosette-forming glioneuronal tumors of the fourth ventricle. J Neuropathol Exp Neurol 73:580-584, 2014

6. Gottfried ON, Fults DW, Townsend JJ, Couldwell WT: Spontaneous hemorrhage associated with a pilomyxoid astrocytoma. Case report. J Neurosurg 99:416-420, 2003

7. Ho CY, Mobley BC, Gordish-Dressman H, VandenBussche CJ, Mason GE, Bornhorst M, et al: A clinicopathologic study of diencephalic pediatric low-grade gliomas with BRAF V600 mutation. Acta Neuropathol 130:575-585, 2015

8. Ishi Y, Yamaguchi S, Yoshida M, Motegi H, Kobayashi H, Terasaka S, et al: Correlation between magnetic resonance imaging characteristics and BRAF alteration status in individuals with optic pathway/hypothalamic pilocytic astrocytomas. J Neuroradiol [epub ahead of print], 2019

9. Jones DT, Gronych J, Lichter P, Witt O, Pfister SM: MAPK pathway activation in pilocytic astrocytoma. Cell Mol Life Sci 69:1799-1811, 2012

10. Jones DT, Hutter B, Jäger N, Korshunov A, Kool M, Warnatz HJ, et al: Recurrent somatic alterations of FGFR1 and NTRK2 in pilocytic astrocytoma. Nat Genet 45:927-932, 2013

11. Jones DT, Kocialkowski S, Liu L, Pearson DM, Bäcklund LM, Ichimura $\mathrm{K}$, et al: Tandem duplication producing a novel oncogenic BRAF fusion gene defines the majority of pilocytic astrocytomas. Cancer Res 68:8673-8677, 2008

12. Karthigeyan M, Singhal P, Salunke P, Vasishta RK: Adult pilomyxoid astrocytoma with hemorrhage in an atypical location. Assian J Neurosurg 14:300-303, 2019

13. Kim MS, Kim SW, Chang CH, Kim OL: Cerebellar pilocytic astrocytomas with spontaneous intratumoral hemorrhage in adult. J Korean Neurosurg Soc 49:363-366, 2011

14. Kitamura Y, Komori T, Shibuya M, Ohara K, Saito Y, Hayashi S, et al: Comprehensive genetic characterization of rosette-forming glioneuronal tumors: independent component analysis by tissue microdissection. Brain Pathol 28:87-93, 2018

15. Lee D, Cho YH, Kang SY, Yoon N, Sung CO, Suh YL: BRAF V600E mutations are frequent in dysembryoplastic neuroepithelial tumors and subependymal giant cell astrocytomas. J Surg Oncol 111:359-364, 2015

16. Linscott LL, Osborn AG, Blaser S, Castillo M, Hewlett RH, Wieselthaler N, et al: Pilomyxoid astrocytoma: expanding the imaging spectrum. AJNR Am J Neuroradiol 29:1861-1866, 2008

17. Lyons MK: Pilocytic astrocytoma with spontaneous intracranial hemorrhages in an elderly adult. Clin Neurol Neurosurg 109:76-80, 2007

18. Ostertun B, Wolf HK, Campos MG, Matus C, Solymosi L, Elger CE, et al: Dysembryoplastic neuroepithelial tumors: MR and CT evaluation. AJNR Am J Neuroradiol 17:419430, 1996

19. Pagano A, Novegno F, Ferlosio A, Lunardi P: Repeat bleeding 18 years after hemorrhagic pilocytic astrocytoma: prognostic implications of conservative management-case report and literature review. World Neurosurg 123:328-338, 2019

20. Qaddoumi I, Orisme W, Wen J, Santiago T, Gupta K, Dalton $\mathrm{JD}$, et al: Genetic alterations in uncommon low-grade neuroepithelial tumors: BRAF, FGFR1, and MYB mutations occur at high frequency and align with morphology. Acta Neuropathol 131:833-845, 2016

21. Schindler G, Capper D, Meyer J, Janzarik W, Omran H, Herold-Mende C, et al: Analysis of BRAF V600E mutation in 1,320 nervous system tumors reveals high mutation frequencies in pleomorphic xanthoastrocytoma, ganglioglioma and extra-cerebellar pilocytic astrocytoma. Acta Neuropathol 121:397-405, 2011

22. Shibahara I, Kanamori M, Kumabe T, Endo H, Sonoda Y, Ogawa Y, et al: Hemorrhagic onset of pilocytic astrocytoma and pilomyxoid astrocytoma. Brain Tumor Pathol 26:1-5, 2009

23. Shibao S, Kimura T, Sasaki H, Fujiwara H, Akiyama T, Ueno $\mathrm{M}$, et al: Hemorrhagic onset of cerebellar pilocytic astrocytoma in an adult: a case report and review of the literature implying a possible relation of degenerative vascular changes to the massive intratumoral hemorrhage. Brain Tumor Pathol 29:96-102, 2012

24. Sun S, Zhou H, Ding ZZ, Shi H: Cerebellar pilocytic astrocytomas with spontaneous intratumoral hemorrhage in the elderly: a case report and review of the literature. Medicine (Baltimore) 97:e11329, 2018

25. Suzuki T, Akamatsu Y, Oshida S, Aso K, Osakabe M, Kashimura H: A case of cisternal pilocytic astrocytoma diagnosed with the balanced steady-state free precession sequence for magnetic resonance imaging: a rare cause of sub- 
arachnoid hemorrhage. World Neurosurg X 1:100003, 2018

26. Thom M, Gomez-Anson B, Revesz T, Harkness W, O'Brien CJ, Kett-White R, et al: Spontaneous intralesional haemorrhage in dysembryoplastic neuroepithelial tumours: a series of five cases. J Neurol Neurosurg Psychiatry 67:97-101, 1999

27. van Baarsen K, Roth J, Serova N, Packer RJ, Shofty B, Thomale UW, et al: Optic pathway-hypothalamic glioma hemorrhage: a series of 9 patients and review of the literature. J Neurosurg 129:1407-1415, 2018

28. White JB, Piepgras DG, Scheithauer BW, Parisi JE: Rate of spontaneous hemorrhage in histologically proven cases of pilocytic astrocytoma. J Neurosurg 108:223-226, 2008

29. Wilson MP, Johnson ES, Hawkins C, Atkins K, Alshaya W, Pugh JA: Hemorrhagic presentations of cerebellar pilocytic astrocytomas in children resulting in death: report of 2 cases. J Neurosurg Pediatr 17:446-452, 2016

30. Yokoyama S, Takayama K, Sueda M, Ishikawa Y, Hirano H: Optic nerve glioma manifesting as intratumoral hemorrhage in a pregnant woman-case report. Neurol Med Chir (Tokyo) 43:559-562, 2003

31. Zhang J, Wu G, Miller CP, Tatevossian RG, Dalton JD, Tang $\mathrm{B}$, et al: Whole-genome sequencing identifies genetic alterations in pediatric low-grade gliomas. Nat Genet 45:602-612, 2013

\section{Disclosures}

The authors report no conflict of interest concerning the materials or methods used in this study or the findings specified in this paper.

\section{Author Contributions}

Conception and design: Ishi. Acquisition of data: Ishi, Okamoto, Motegi, Terasaka. Analysis and interpretation of data: Ishi, Hatanaka. Drafting the article: Ishi. Critically revising the article: Yamaguchi, Hatanaka. Reviewed submitted version of manuscript: Yamaguchi. Approved the final version of the manuscript on behalf of all authors: Yamaguchi. Statistical analysis: Ishi. Administrative/technical/material support: Hatanaka, Okamoto, Motegi, Kobayashi, Terasaka. Study supervision: Houkin.

\section{Correspondence}

Shigeru Yamaguchi: Hokkaido University Graduate School of Medicine, Sapporo, Japan. yama-shu@med.hokudai.ac.jp. 